\title{
Quality lines
}

doi:10.1136/qshc.2009.035477

David P Stevens, Editor-in-Chief

\section{Fresh perspectives on handover research}

Handovers in physician postgraduate training continue to be a troubling source of error. This is highlighted by fresh perspectives in this issue of OSHC provided by five reports and a commentary from the US, UK, and Australia. Scholars in both the US and UK emphasize that effective handovers must be mastered systematically as a competency. All highlight the role of professional responsibility in assuring the safe, successful and informative handover. In one of the US studies, less than twothirds of the combined oral and written sign-outs describe the patient's clinical condition, hospital course, and whether or not there was a task to be completed by the covering physician. While the content of the handover has been a focus of many studies, the active involvement of the clinician who receives the handover is highlighted by several of these reports. For example, one study found that only one in five asked questions. The presence or absence of "trust in the source" is highlighted as a key element by one author. The authors of these reports conclude that systems changes such as team sign-out, templated written sign-outs and reduction of serial sign-outs in the same day have the potential to improve handoff safety and quality. An accompanying commentary proposes that the research agenda suggested by these papers should focus on handover content, institutional culture, and professional competence.

See pages 244, 248, 256, 261, 267, 272

\section{Improving the improvement of medical education}

Two mixed-methods studies from a US medical school focus on modelling institutional improvement as an essential characteristic of school administration for medical schools that seek to instil knowledge for healthcare improvement in their graduates. One study provides an example of a process of improvement of the Medical Student Performance Evaluation (MSPE)

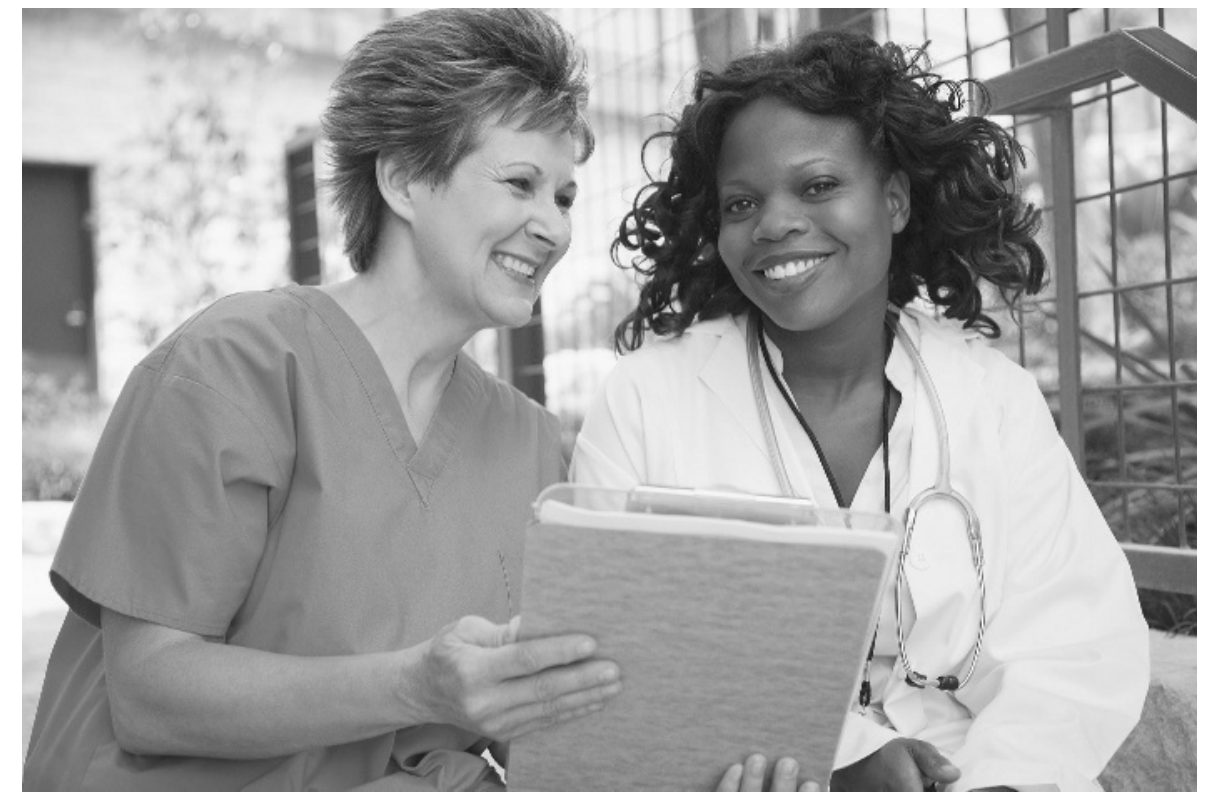

letter, the summary document that US medical schools send to prospective postgraduate training sites. By applying continuous improvement principles to the construction of the MSPE the overall efficiency of the process was enhanced, and the MSPE committee was able to spend less time on structure and format, and more on the content of the letters. A second report describes development of a method to monitor and analyze patient encounters in the third year of medical school. This educational improvement project allowed course directors to (1) confirm adequate clinical exposure, (2) obtain prompt information on student experiences, (3) adjust individual student rotations to meet requirements and (4) ascertain the range of clinical experiences available to students. An accompanying commentary highlights the research agenda that can be advanced by modelling quality improvement in settings where health professional students learn. See pages 246, 278 and 283

Root Cause Analysis in the UK: the challenge of maintaining skill The National Patient Safety Agency (NPSA) for England and Wales in 2004-5 undertook an ambitious training programme for NHS trusts in patient safety investigation, using Root Cause Analysis (RCA). The NPSA trained over 7000 staff in two years, covering the 607 trusts in England and Wales. A survey evaluation showed that healthcare staff believed their ability to conduct RCA was high immediately after attending the programme. Their knowledge applied to specific practical scenarios was more variable, suggesting some needed more direct feedback on the application of RCA than was available via the workshops. However, they also experienced problems in conducting RCA as taught, and a sample assessed six months after the course were also concerned about the extent their RCA reports were acted upon. The results are similar to those found in Australia, and indicate that trusts need to invest in systems for maintaining the skill base of RCA investigators, provide feedback and higher level training, and use quality improvement methods to ensure learning is achieved and implemented within the wider healthcare economy.

See page 288 